\title{
In melanoma ulceration, size matters
}

Numerous studies have assessed the prognostic value of the size of ulceration in clinically localized cutaneous melanoma. In fact, the presence of ulceration was already established as an adverse prognostic factor back in the 1950s. Now, the team led by John Thompson and Richard Scolyer has not only confirmed the importance of the extent of ulceration in predicting melanoma prognosis, but has also described how the size of the ulceration is actually a better prognosis factor than the mere presence or absence of ulceration.

The study included 521 patients with ulcerated melanoma and 4,140 patients with non-ulcerated melanoma. The patients were then distributed into different categories: patients with absence or presence of ulceration; minimally/ moderately ulcerated disease (in whom the percentage of ulceration was $\leq 70 \%$ or $\leq 5 \mathrm{~mm}, n=163$ and 388 respectively); and extensive ulcerated tumours (in whom the extent of the ulceration was $>70 \%$ or $>5 \mathrm{~mm}, n=54$ and 133 respectively). The 10-year melanoma-specific survival was superior in patients with non-ulcerated melanoma (81\%), compared with patients with ulcerations (62\%). Among the patients with ulceration, 10-year melanoma-specific survival in patients with minimally/ moderately ulcerated disease was considerably higher (approximately 68\%) than in patients with extensive ulcerated disease (approximately 35\%), confirming that stratifying ulceration provides a more accurate prognosis than the presence of ulceration alone.

Because the gene profiles of ulcerated and non-ulcerated melanomas are very different, further studies would confirm if these genetic profiles could explain the different behaviour of the disease.

\section{Teresa Villanueva}

Original article In 't Hout, F. E. et al. Prognostic importance of the extent of ulceration in patients with clinically localized cutaneous melanoma. Ann. Surg. doi:10.1097/SLA.0b013e31824c4b0b 